\title{
Gutenberg-Richter statistics in topologically realistic system-level earthquake stress-evolution simulations
}

\author{
John B. Rundle ${ }^{1}$, Paul B. Rundle ${ }^{2}$, Andrea Donnellan ${ }^{3}$, and Geoffrey Fox ${ }^{4}$ \\ ${ }^{1}$ Center for Computational Science and Engineering, University of California, Davis, Davis, CA 95616; and Distinguished Visiting Scientist, \\ Earth \& Space Sciences Division, Jet Propulsion Laboratory, Pasadena, CA 91125, U.S.A \\ ${ }^{2}$ Department of Physics, Harvey Mudd College, Claremont, CA, U.S.A. \\ ${ }^{3}$ Earth \& Space Sciences Division, Jet Propulsion Laboratory, Pasadena, CA, U.S.A. \\ ${ }^{4}$ Department of Computer Science, Indiana University, Bloomington, IN, U.S.A.
}

(Received December 1, 2003; Revised May 6, 2004; Accepted July 1, 2004)

We discuss the problem of earthquake forecasting in the context of new models for the dynamics based on statistical physics. Here we focus on new, topologically realistic system-level approaches to the modeling of earthquake faults. We show that the frictional failure physics of earthquakes in these complex, topologically realistic models leads to self-organization of the statistical dynamics, and produces statistical distributions characterizing the activity, notably the Gutenberg-Richter magnitude frequency distribution, that are similar to those observed in nature. In particular, we show that a parameterization of friction that includes a simple representation of a dynamic stress intensity factor is needed to organize the dynamics. We also show that the slip distributions for synthetic events obtained in the model are also similar to those observed in nature

Key words: Earthquakes, simulations, forecasting, stress, interactions, complex systems, scaling, systems.

\section{Introduction}

Earthquakes have great scientific, societal, and economic significance. During the first three months of 2001, the January 13, 2001 magnitude 7.6 El Salvador earthquake, the January 26, magnitude 7.9 Gujarat, India earthquake, and the February 28, 2001 magnitude 6.8 Seattle, Washington, USA event killed thousands of persons and caused billions of dollars in property losses. The January 16, 1995 Kobe, Japan earthquake was only a magnitude 6.9 event and yet produced an estimated $\$ 200$ billion loss. Despite an active earthquake forecasting/prediction program in Japan, this event was a complete surprise. Similar scenarios are possible in Los Angeles, San Francisco, Seattle, and other urban centers around the Pacific plate boundary.

The magnitude of the potential loss of life and property in earthquakes is so great that reliable earthquake forecasting has been a long-sought goal. Examples of recent large earthquakes affecting life and property include the January 13, 2001 magnitude 7.6 El Salvador earthquake, the January 26, magnitude 7.9 Gujarat, India earthquake, and the February 28, 2001 magnitude 6.8 Seattle, Washington, USA event. Many millions of dollars and many thousands of work years have been spent on observational programs searching for reliable precursory phenomena. Possible precursory phenomena include changes in seismicity, changes in seismic velocities, tilt and strain precursors, electromagnetic signals, hydrologic phenomena, and chemical emissions (Scholz, 1990; Turcotte, 1991). A few successes have been reported, but to

Copy right (c) The Society of Geomagnetism and Earth, Planetary and Space Sciences (SGEPSS); The Seismological Society of Japan; The Volcanological Society of Japan; The Geodetic Society of Japan; The Japanese Society for Planetary Sciences; TERRAPUB. date, no precursors to large earthquakes have been detected that would provide reliable forecasts (Nature, 1999).

In terms of data acquisition several major approaches are currently being emphasized. These include:

1. Paleoseismic observations of historic earthquakes whose occurrence and locations are preserved in offset surface sediments;

2. Patterns of seismicity (origin time, location, magnitude of earthquakes);

3. Surface deformation measured via Global Positioning System (GPS) networks such as the Southern California Integrated GPS Network (SCIGN), and the Bay Area Regional Deformation (BARD) network (SCEC; Nature, 1999).

4. Synthetic Aperture Radar Interferometry (InSAR) observations of surface displacement. Observations of these data types are also planned as part of the Earthscope NSF/GEO/EAR/MRE initiative. In fact, the Plate Boundary Observatory (PBO) plans to place more than a thousand GPS, strainmeter, and deformation sensors along the active plate boundary of the western coast of the United States, Mexico and Canada, at an eventual cost in excess of $\$ 100$ million (Nature, 1999).

It is clearly a very high priority to utilize this wealth of new data to better understand the fundamentals of earthquake occurrence. This understanding can improve several aspects of the earthquake hazard. For example:

1. Risk assessment. Determining the probability of the occurrence of an earthquake of a specified magnitude 
in a specified area within a specified time window.

2. Earthquake forecasting (prediction). Finding patterns of behavior that can provide statistically acceptable forecasts of future major earthquakes.

\section{Earthquakes}

\subsection{Numerical simulations}

Earthquakes are a complex nonlinear dynamical system, so that techniques appropriate for the study of linear systems have not been of much use. There are two serious drawbacks to a purely observational approach to the problem of earthquake forecasting: 1) Inaccessible and unobservable stressstrain dynamics, and 2) Multiscale dynamics that cover a vast range of space and time scales. Because of these fundamental problems, the use of numerical simulations, together with theory and analysis, is mandatory if we are to discover answers to the questions above. Correspondingly, all types of earthquake-related data, including seismic, geodetic, paleoseismic, and laboratory rock mechanics experiments must be employed. The data are used both to determine physical properties of the models we simulate, a process of data assimilation, as well as to critically test the results of our simulation-derived hypotheses, so that future hypotheses can be developed. Several authors have pursued numerical simulations of this type (Rundle, 1988; Ward, 2000; Hashimoto, 2001; Rundle et al., 2001).

\subsection{Unobservable dynamics}

Geologic observations indicate that earthquake faults occur in topologically complex, multi-scale networks that are driven to failure by external forces arising from plate tectonic motions (Rundle et al., 2000a, 2001; Ward, 2000). The basic problem in this class of systems is that the true stress-strain dynamics is inaccessible to direct observations, or unobservable. For example, the best current compendium of stress magnitudes and directions in the earth's crust is the World Stress Map (Zoback, 1992), entries on which represent point static time-averaged estimates of maximum and minimum principal stresses in space. Since to define the fault dynamics, one needs dynamic stresses and strains for all space and time, the WSM data will not be sufficient for this purpose.

Conversely, the space time patterns associated with the time, location, and magnitude of the earthquakes are easily observable. Our scientific focus is therefore on understanding how the observable space-time earthquake patterns are related to the fundamentally inaccessible and unobservable dynamics, thus we are developing new data-mining, pattern recognition, theoretical analysis and ensemble forecasting techniques. In view of the lack of direct observational data, any new techniques that use space-time patterns of earthquakes to interpret underlying dynamics and forecast future activity must be developed via knowledge acquisition and knowledge reasoning techniques derived from the integration of diverse and indirect observations, combined with a spectrum of increasingly detailed and realistic numerical simulations of candidate models.

\subsection{Multiscale dynamics}

The second problem is that earthquake dynamics are strongly coupled across a vast range of space and time scales that are both much smaller and much larger than "human" dimensions (GEM; ACES; SCEC; Mora, 1999; Matsu'ura et al., 2001). The important spatial scales span the range from the grain scale, of $1 \mathrm{~nm}$ to $1 \mathrm{~cm}$; the fault zone scale, at $1 \mathrm{~cm}$ to $100 \mathrm{~m}$; the fault segment scale, at $100 \mathrm{~m}$ to 10 $\mathrm{km}$; the fault system or network scale, at $10 \mathrm{~km}$ to 1000 $\mathrm{km}$; finally to the Tectonic plate boundary scale in excess of $1000 \mathrm{~km}$. Important time scales span the range from the source process time scale of fractions of seconds to seconds; to the stress transfer scale of seconds to years; to event recurrence time scales of years to many thousands of years; finally to the fault topology evolution scale, in excess of many thousands of years up to millions of years. There is considerable evidence that many/most/all of these spatial and temporal scales are strongly coupled by the dynamics. Consider, as evidence, the Gutenberg-Richter relation, which is a power law for frequency of events in terms of cumulative event sizes. Power laws are a fundamental property of scaleinvariant, self-organizing systems (Vicsek, 1989; Gouyet, 1996) whose dynamics and structures are strongly coupled and correlated across many scales in space and time. If the dynamics were instead unconnected or random, one would expect to see Gaussian or Poisson statistics.

Simulations can help us to understand how processes operating on time scales of seconds and spatial scales of meters, such as source process times in fault zones, influence processes that are observed to occur over time scales of hundreds of years and spatial scales of hundreds of kilometers, such as recurrence of great earthquakes. Numerical simulations also allow us to connect observable surface data to underlying unobservable stress-strain dynamics, so we can determine how these are related. Thus we conclude that numerical simulations are mandatory if we are to understand the physics of earthquake fault systems.

\section{The Virtual California Model}

Although all scales are important, we place more emphasis on the fault system or fault network scale, since this is the scale of most current and planned observational data networks. It is also the scale upon which the data we are interested in understanding, large and great earthquakes, occur. Furthermore, since it is not possible to uniquely determine the stress distribution on the southern California fault system, and since the friction laws and elastic stress transfer moduli are not known, it makes little sense to pursue a deterministic computation to model the space-time evolution of stress on the fault system. We therefore coarse-grain over times shorter than the source process time, which means we either neglect wave-mediated stress transfer, or we represent it in simple ways.

The Virtual California model (Rundle et al., 2000a, b, 2001) is a stochastic, cellular automata instantiation of an earthquake backslip model, in that loading of each fault segment occurs via the accumulation of slip deficit $\phi(x, t)=$ $s(\boldsymbol{x}, t)-V t$, where $s(\boldsymbol{x}, t)$ is slip, $V$ is long term slip rate, and $t$ is time. Basic details of how the model is constructed, how physical properties such as friction coefficients are computed using historical earthquakes and then assigned to fault segments, and how slip is adjusted during earthquakes are given in Rundle et al. (2000b). At the present time, faults used in the model are exclusively vertical strike slip faults, 
the most active faults in California, and upon which most of the seismic moment release is localized. Thrust earthquakes, such as the 1994 Northridge and 1971 San Fernando faults, are certainly damaging, but they occur infrequently and are therefore regarded as perturbations on the primary strike slip fault structures.

An important fact to recognize is that the data inputs to Virtual California are temporally- or spatially-averaged parameters, such as average recurrence intervals for fault segments, and average slip magnitudes. However, an important output is the variability in time and space of these same physical parameters. These output statistics are a consequence of the dynamics, the stress interactions, and the dataderived model parameters. Therefore the variability in the output statistics and the associated probability density functions represent an important independent product of the simulation that can be compared to variability in observations to obtain further insight. Corresponding observations of natural variability on fault systems are discussed in Stein and Newman (2004).

The Virtual California model also has the following additional characteristics.

1. Surfaces of discontinuity (faults) across which slip is discontinuous at the time of an earthquake, and which are subject to frictional resistance. Here we restrict the model to only topologically complex systems of vertically dipping faults mirroring the complexity found on the natural fault networks of southern California.

2. Stochastic dynamics. In these models, we are interested in the space-time patterns and correlations that emerge from the underlying stress-strain dynamics. These correlations evolve over many hundreds or thousands of years, time scales much longer than the time scales associated either with rupture or elastic wave periods. Most of the elastic and frictional parameters for faults and earth materials, although known in the laboratory, will likely remain poorly defined in nature. For this reason, it makes little sense to attempt a deterministic solution to the equations of motion. Instead, we use a Cellular Automaton (CA) approach, in which the dynamics is parameterized by random variables chosen from well defined probability distributions. The stochastic nature of the dynamics is implemented during the sliding process, in which, to the computed slip of a fault segment to reduce its stress, a random overshoot or undershoot of $\pm 10 \%$ is added. The density function characterizing the overshootundershoot is a uniform probability density function.

3. Linear elastic stress transfer or interactions between fault surfaces. Again, although most of the significant parameters associated with rupture, such as friction coefficients and friction law constants and functions can be defined and measured in the laboratory, current experience indicates they will likely always be poorly known for faults in nature. We use quasistatic stress interaction (Green's function) tensors $T_{i j}^{k l}\left(\boldsymbol{x}-\boldsymbol{x}^{\prime}\right)$, which we will write henceforth schematically as $T\left(\boldsymbol{x}-\boldsymbol{x}^{\prime}\right)$, since it has been shown that in mean field elastic systems the order of failing sites is unimportant (Preston, 2001). Signal propagation due to elastic waves is therefore not necessary to establish the ordering of sites that fail.

4. Persistent increase of stresses on the fault surfaces arising from plate tectonic forcing parameterized via the backslip method. This method has the advantage that it matches the long term rate of offset $V$ in model faults with the geologically known long term slip rate on faults in nature. Stress increase occurs via the following physics. The stress tensor $\sigma_{i j}(\boldsymbol{x}, t)$ is related to the slip $s_{l}(\boldsymbol{x}, t)$ by:

$$
\sigma_{i j}(\boldsymbol{x}, t)=\int d \boldsymbol{x}_{k} T_{i j}^{k l}\left(\boldsymbol{x}-\boldsymbol{x}^{\prime}\right) s_{l}\left(\boldsymbol{x}^{\prime}, t\right) .
$$

Now if $\boldsymbol{x}=\boldsymbol{x}^{\prime}$, a positive $\operatorname{slip} s_{l}(\boldsymbol{x}, t)>0$ results in a decrease in stress, $\Delta \sigma_{i j}(\boldsymbol{x}, t)<0$. Therefore, if we write the equation:

$$
\sigma_{i j}(\boldsymbol{x}, t)=\int d \boldsymbol{x}_{k} T_{i j}^{k l}\left(\boldsymbol{x}-\boldsymbol{x}^{\prime}\right)\left\{s_{l}\left(\boldsymbol{x}^{\prime}, t\right)-V_{l}\left(\boldsymbol{x}^{\prime}\right) t\right\}
$$

where $V_{l}(\boldsymbol{x}) t=\left\langle s_{l}(\boldsymbol{x}, t)\right\rangle$ is the average long term rate of slip at $\boldsymbol{x}^{\prime}$ over time interval $t$, then the second term $-V_{l}(\boldsymbol{x}) t$ leads to an increase in the stress, $\Delta \sigma_{i j}(\boldsymbol{x}, t)>0$. Therefore the second term is the stress accumulation term.

In applying Eqs. (1) and (2) in our simulations, it should be noted that we use the discrete form of the integrals, although we continue to use the continuous (integral) form of the equations in this paper for notational simplicity. In the discrete form, the continuous fault surface is replaced by a network of 650 rectangular segments, each about $10 \mathrm{~km}$ in length along strike, with a uniform depth of $15 \mathrm{~km}$. The stress Greens functions, or stress transfer coefficients for the influence of segment $i$ upon segment $j$ are computed by imposing a unit $(1 \mathrm{~m})$ of slip on segment $i$, then computing the change in "average" shear and normal stress on segment $j$. "Average" in this sense means that we subdivide segment $j$ into $6 \times 6=36$ sub-segments, then we compute the stress change due to slip on segment $i$ on each of these sub-segments, then we average the result.

5. Parameters for friction laws and fault topology that are determined by assimilating seismic, paleoseismic, geodetic, and other geophysical data from events occurring over the last $\sim 200$ years in California (Rundle et al., 2000b, 2001; see below for discussion).

6. Frictional resistance laws (Rabinowicz, 1995) that range from the simplest Amontons-Coulomb stick-slip friction, to heuristic laws such as slip- or stress rate dependent weakening laws based on recent laboratory friction (Tullis, 1996) and fracture experiments (Kanninen and Popelar, 1985; Freund, 1990; Saxena, 1998). These laws are related to rate-and-state and leaky threshold laws (Rundle et al., 2001).

In general, several of the friction laws described above can be written in the following representative, equivalent forms on an element of fault surface:

$$
\begin{aligned}
& \frac{\partial \sigma}{\partial t}=K_{L} V-f(\sigma, V) \\
& K_{L} \frac{\partial s}{\partial t}=f(\sigma, V) .
\end{aligned}
$$

Here $s(\boldsymbol{x}, t)$ is slip at position $\boldsymbol{x}$ and time $t, \sigma(\boldsymbol{x}, t)$ is shear stress, $K_{L}$ is the self-interaction or "stress drop stiffness" and $f[\sigma, V]$ is the stress dissipation function (Rundle et al., 2001, 2002). For example, the "Amontons" or Coulomb friction law, having a sharp failure threshold, can be written 
in the form (2) using a Dirac delta function:

$$
\frac{\partial s}{\partial t}=\frac{\Delta \sigma}{K_{L}} \delta\left(t-t_{F}\right)
$$

where the stress drop $\Delta \sigma=\sigma-\sigma^{R}(V)$ and $\sigma^{R}(V)$ is the velocity-dependent residual stress. For laboratory experiments, $K_{L}$ is the \{machine + sample $\}$ stiffness, and for simulations, $K_{L}$ represents the stiffness of a coarse-grained element of the fault of scale size $L . \delta()$ is the Dirac delta, and $t_{F}$ is any time at which $\sigma\left(\boldsymbol{x}, t_{F}\right)=\sigma^{F}(V)$. Both $\sigma^{F}$ and $\sigma^{R}$ can also be parameterized as functions of the normal stress $\chi$ by means of coefficients of static $\mu_{S}$ and ("effective") kinetic $\mu_{K}$ coefficients of friction, $\sigma^{F}=\mu_{S} \chi, \sigma^{R}=\mu_{K} \chi$.

The method for data assimilation used is explained in more detail in Rundle et al. (2000b). Briefly, we take the moment released by large earthquakes in the historic record over the last 200 years or so and assign it, using a $1 / r_{i j}^{3}$ probability density function, to all of the fault segments in the system. Here $r_{i j}=\left|\boldsymbol{x}_{i}-\boldsymbol{x}_{j}\right|$ is the distance of the earthquake with epicenter at $\boldsymbol{x}_{i}$ to the fault segment centered at $\boldsymbol{x}_{j}$. This procedure leads to the assignment of an average, characteristic seismic moment to each fault segment. Once the moment is assigned, we use the slip:stress-drop relation for each rectangular fault segment, computed from the discrete equation corresponding to (1) to obtain the average stress drop at failure $\Delta \sigma_{i}^{F}$ for the segment. Then, given a nominal normal stress across the fault segment due to gravity stresses $N_{g}\left(\boldsymbol{x}_{i}\right)$, we compute the difference between the static and kinetic frictional coefficients $\Delta \sigma_{i}^{F} \equiv\left(\mu_{S}-\mu_{K}\right)_{i} N_{g}\left(\boldsymbol{x}_{i}\right)$. However, in applying the slip dynamics of the model, we compute the slip needed to relieve the stress drop $\Delta \sigma_{i}^{F}$ due to both the gravitational normal stress $N_{g}\left(\boldsymbol{x}_{i}\right)$ as well as all elastic contributions $N_{e}\left(\boldsymbol{x}_{i}\right)$ to the total space- and time-dependent normal stress $\chi\left(\boldsymbol{x}_{i}, t\right)=N_{g}\left(\boldsymbol{x}_{i}\right)+N_{e}\left(\boldsymbol{x}_{i}, t\right)$.

In recent work (Rundle et al., 2001), we have introduced another parameter $\alpha$, which allows for stable stressdependent aseismic sliding. The process described by $\alpha$ is seen in laboratory friction experiments (Tullis, 1996), and is expressed by a generalization of Eq. (4):

$$
\frac{\partial s}{\partial t}=\frac{\Delta \sigma}{K_{L}}\left\{\alpha+\delta\left(t-t_{F}\right)\right\}
$$

We found that the parameter $\alpha$, which can be fixed either through laboratory experiments or through field observations (Tullis, 1996; Deng and Sykes, 1997), acts to smooth the stress field a fault when $\alpha>0$, and to roughen the fault stress field when $\alpha<0$. In Virtual California, the value of $\alpha$ for each segment, $\alpha_{i}$, is assigned for each segment based upon field observations, since it can be shown that $\alpha$ is equal to the ratio of aseismic slip to total slip (seismic + aseismic) during an average slip cycle on a segment. Also, laboratory observations (Tullis et al., 1996; Karner and Marone, 2000) indicate that observed values of $\alpha$ are a few percent for sliding of granite on granite, so where no observations exist, we assume that all segments have a minimum value of $\alpha \approx$ 1 .

In the model results that we describe here, we further generalize (5) to include an additional term which depends on rate of stress increase:

$$
\frac{\partial s}{\partial t}=\frac{\Delta \sigma}{K_{T}}\left\{\alpha+\delta\left(t-t_{F}\right)+\beta \sigma\left(\frac{\partial \sigma}{\partial t}-\eta\right)\right\} .
$$

Here $\beta$ is a constant having appropriate units (stress/time ${ }^{2}$ ), $\eta$ is a critical ("dynamic") stressing rate, and $K_{T}$ represents the total spring constant associated with a fault segment. The last term can be considered to be parameterization of effects associated with a dynamic stress intensity factor (Kanninen and Popelar, 1985; Freund, 1990; Saxena, 1998). It is known that stress rate effects are important in the process of dynamic fracture, such as might be expected during an earthquake. For example, the stress intensity factor $K_{I}$ for mode $I$ tensile fracture is thought to be of the form:

$$
K_{I D}=K_{I D}\left(\frac{\partial \sigma}{\partial t}, T\right)
$$

where $T$ is temperature. More specifically, for a crack propagating at velocity $v$, it has been proposed that the time dependent dynamic stress intensity factor $K_{D}(t)$ is of the general form (Kanninen and Popelar, 1985):

$$
K_{D}(t)=k(v) K_{D}(0)=k(v) K_{S}
$$

where $K_{S}$ is the static stress intensity factor. While not of the exact form of either Eq. (7) or (8), Eq. (6) is an expression of the idea that the onset of earthquake sliding depends on the stressing rate through a critical threshold value $\eta$.

In the simulations described below, we implement the physical process described by Eq. (6) in our Virtual California CA simulations as follows. We define the Coulomb Failure Function $C F F(\boldsymbol{x}, t)$ :

$$
C F F(\boldsymbol{x}, t)=\sigma(\boldsymbol{x}, t)-\mu_{S} \chi(\boldsymbol{x}, t) .
$$

According to the first term in Eq. (6), stable slip can occur with amplitude proportional to $\alpha$ for nonzero $\Delta \sigma$. In addition, according to the second term, unstable failure of a fault always occurs when $C F F(\boldsymbol{x}, t)=0$. To implement a failure mechanism in a simple way that demonstrates physics similar to the third term, we allow unstable slip of amplitude:

$$
\frac{\Delta \sigma}{K_{T}}=\frac{\sigma(\boldsymbol{x}, t)-\mu_{K} \chi(\boldsymbol{x}, t)}{K_{T}}
$$

when the condition:

$$
-\frac{\partial}{\partial t} \log \{-C F F(\boldsymbol{x}, t)\}>\frac{\eta}{\Delta \sigma^{F}}
$$

is met. Here $\Delta \sigma_{i}^{F} \equiv\left(\mu_{S}-\mu_{K}\right)_{i} \chi\left(\boldsymbol{x}_{i}\right)$. We set:

$$
\eta \equiv \frac{\epsilon \Delta \sigma^{F}}{\Delta t}
$$

where $\Delta t$ is the time step in the simulation, and $\epsilon$ is a chosen parameter $0<\epsilon<1$. For a discrete time step $\Delta t$ as is used in CA computations, (11) is implemented as:

$$
\frac{C F F(\boldsymbol{x}, t)-C F F(\boldsymbol{x}, t+\delta t)}{C F F(\boldsymbol{x}, t)}>\frac{\eta \Delta t}{\Delta \sigma^{F}}
$$




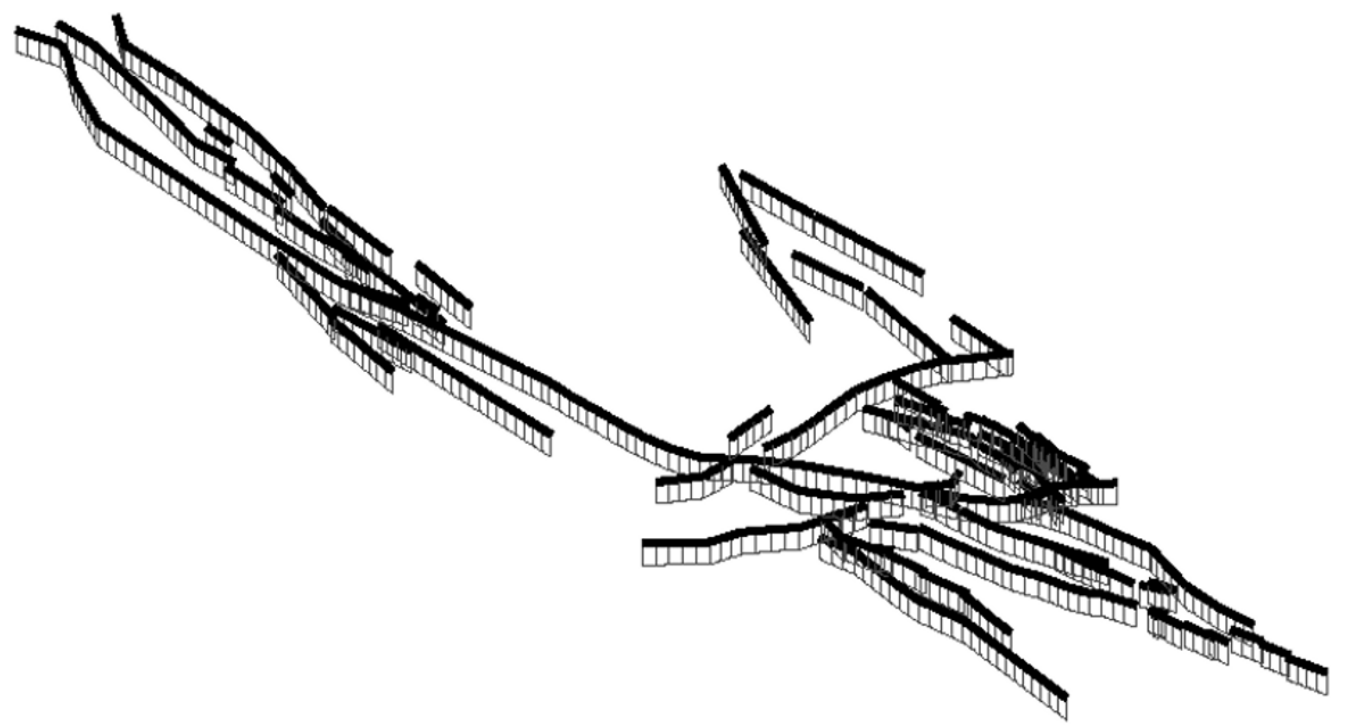

Fig. 1. Faults segments making up the Virtual California model. Model has 650 fault segments, each approximately $10 \mathrm{~km}$ in length along strike, and exactly $15 \mathrm{~km}$ in depth.

(recall that $C F F(\boldsymbol{x}, t) \geq 0$ ). In Eq. (13), we interpret $\delta t$ as being the time since the beginning of the earthquake at time $t$. Implicitly, it is assumed in (6), (11) and (13) that:

$$
\left.\eta \gg \frac{\delta \sigma(\boldsymbol{x}, t)}{\delta t}\right|_{\text {Interseismic }}=-\int d \boldsymbol{x}_{k} T_{i j}^{k l}\left(\boldsymbol{x}-\boldsymbol{x}^{\prime}\right) V_{l}\left(\boldsymbol{x}^{\prime}\right)
$$

i.e., that the $\eta$-value for stress-rate triggering is much larger the stress rate characterizing interseismic stress accumulation.

As described above, the Virtual California simulation uses a cellular automaton (CA) dynamics to evolve the stress field in response to the persistently increasing stress due to the "backslip" on the fault. In other words, because Eq. (1) represents a decrease in stress at $\boldsymbol{x}$ in response to slip at $\boldsymbol{x}$, the part of Eq. (2) defined by:

$$
\sigma_{i j}(\boldsymbol{x}, t)=-\int d \boldsymbol{x}_{k} T_{i j}^{k l}\left(\boldsymbol{x}-\boldsymbol{x}^{\prime}\right) V_{l}\left(\boldsymbol{x}^{\prime}\right) t
$$

represents an increase in stress at $\boldsymbol{x}$ in response to the long term (plate tectonic) loading $V_{l}(\boldsymbol{x}) t$. In the CA approach, the code steps through time $t$ in steps of size $\Delta t$ on the loading time scale. We choose these time steps to be "small" but exactly how small depends to some extent on the application of interest. Usually, we use time steps $\Delta t=1$ year, but in cases in which we want to be very sure that there is a high probability of having at most 1 earthquake on each time step (rather than 2 or 3 , for example). To make this property more transparent, we keep count of the average number of initiator sites, i.e., sites having $C F F(x, t) \geq 0$ just after a time update step $\Delta t$ has been applied, and also the number of such sites on that time step. With a sample simulation, we can judge how small to fix $\Delta t$ so that, with $95 \%$ confidence, there will be only one initiator (thus only one earthquake) per time step. For example, to tabulate the Gutenberg-Richter statistics shown below, we used $\Delta t=.2$ year. By contrast, for $\Delta t=1$ year, which is a time step we typically use in general stress evolution analyses, we find that there is roughly 1 initiator site per time step with roughly $66 \%$ confidence. Note that, during these loading time steps, a small amount of stable slip $\Delta s=\alpha\left(\sigma-\sigma^{R}\right)$ occurs due to the stress leakage process described by $\alpha$ in Eqs. (5) and (6). These slip increments are applied just after a loading update has occurred.

We continue stepping through time until $C F F(\boldsymbol{x}, t) \geq 0$ is established on at least one site, at which time we fix $t$ and proceed to the stochastic slip adjustment procedure. In this procedure, we proceed in a series of parallel monte carlo sweeps (mcs). On the first mcs, all failing sites having the condition $C F F(\boldsymbol{x}, t) \geq 0$ are adjusted by a slip amount:

$$
\Delta s=\frac{\left\{\sigma-\sigma^{R}\right\}}{K_{T}}(1+\rho)
$$

where $\rho$ is a random variable, typically having a uniform probability density function on the interval $(-.1, .1)$, to model random overshoot or undershoot. After the initiator site(s) have been "slipped" or "failed", shear and normal stress are transferred to all other sites using the discrete form of the stress Green's functions or stress transfer coefficients $T_{i j}^{k l}\left(\boldsymbol{x}-\boldsymbol{x}^{\prime}\right)$. At this point, the second mcs begins, and any other site now having the stress magnitude condition $C F F(x, t) \geq 0$, or the stress rate condition described by (13), is failed as well, their stress is redistributed using $T_{i j}^{k l}\left(\boldsymbol{x}-\boldsymbol{x}^{\prime}\right)$, and so forth. In general, no healing of the segments is permitted until the end of the slip adjustment process. At the conclusion of the mcs slip adjustments, the loading process is resumed by stepping $t$ by $\Delta t$ and so on.

\section{Results and Conclusions}

Fault Model. The fault model we used in the Virtual California simulations described here is shown in Fig. 1. It is a far more detailed representation of the faults used for the southern California model described in earlier work (Rundle et al., 2001). The geometry of most of southern California is based upon Table 2 of Deng 
Table 1. Table of fault data for the Virtual California model used in this paper. Each fault segment is approximately $10 \mathrm{~km}$ in length along strike, and 15 $\mathrm{km}$ in depth. "Chart Distance" refers to plots such as that of Fig. 2, in which all segments are concatenated end-to-end for plotting purposes. For slip rates, positive slip rate is right lateral, negative slip rate is left lateral.

\begin{tabular}{|c|c|c|c|c|c|}
\hline \multirow{2}{*}{$\begin{array}{l}\text { Fault or Fault } \\
\text { System Name }\end{array}$} & \multicolumn{2}{|c|}{ Segment Nos. } & \multicolumn{2}{|c|}{ Chart Distance $(\mathrm{km})$} & \multirow[t]{2}{*}{ Average Slip Rate (mm/yr) } \\
\hline & Begin & End & Begin & End & \\
\hline Bartlett Springs & 0 & 7 & 0.0 & 84.7 & 6 \\
\hline Calaveras & 8 & 22 & 84.7 & 238.9 & $15(8->17) 6(18->22)$ \\
\hline Collayomi & 23 & 25 & 238.9 & 266.8 & .6 \\
\hline Concord-Green Valley & 26 & 31 & 266.8 & 322.2 & 6 \\
\hline Death Valley & 32 & 55 & 322.2 & 569.6 & $5(32->49) 4(50->55)$ \\
\hline Garberville-Briceland & 56 & 59 & 569.6 & 609.2 & 9 \\
\hline Greenville & 60 & 66 & 609.2 & 682.2 & 2 \\
\hline Hayward & 67 & 77 & 682.2 & 793.3 & $9(67->74) 3(75->77)$ \\
\hline Hunter Mtn.-Saline Val. & 78 & 84 & 793.3 & 861.3 & 2.5 \\
\hline Hunting Creek-Berryessa & 85 & 90 & 861.3 & 920.3 & 6 \\
\hline Lake Mountain & 91 & 93 & 920.3 & 953.7 & 6 \\
\hline Maacama & 94 & 111 & 953.7 & 1133.3 & 9 \\
\hline Monterey Bay-Tularcitos & 112 & 119 & 1133.3 & 1213.6 & .5 \\
\hline Ortigalita & 120 & 126 & 1213.6 & 1280.1 & 1 \\
\hline Owens Valley & 127 & 138 & 1280.1 & 1401.6 & 1.5 \\
\hline Palo Colorado-Sur & 139 & 146 & 1401.6 & 1479.8 & 3 \\
\hline Panamint Valley & 147 & 156 & 1479.8 & 1584.5 & 2.5 \\
\hline Quien Sabe & 157 & 158 & 1584.5 & 1607.6 & 1 \\
\hline Rinconada & 159 & 177 & 1607.6 & 1796.9 & 1 \\
\hline Rodgers Creek & 178 & 183 & 1796.9 & 1858.9 & 9 \\
\hline Round Valley & 184 & 189 & 1858.9 & 1914.3 & 6 \\
\hline San Gregorio & 190 & 198 & 1914.3 & 2003.3 & 5 \\
\hline Sargent & 199 & 203 & 2003.3 & 2056.0 & 3 \\
\hline West Napa & 204 & 206 & 2056.0 & 2085.9 & 1 \\
\hline White Mountains & 207 & 216 & 2085.9 & 2186.5 & 1 \\
\hline San Andreas North & 217 & 263 & 2186.5 & 2653.6 & $24(217->248) 17(249->263)$ \\
\hline San Andreas Creeping & 264 & 273 & 2653.6 & 2751.3 & 34 \\
\hline San Andreas South & 274 & 335 & 2751.3 & 3330.7 & $34(274->298) 30(299->312)$ \\
\hline & & & & & $24(313->321) 25(322->335)$ \\
\hline San Jacinto & 336 & 364 & 3330.7 & 3622.1 & $12(336->352) 14(353->364)$ \\
\hline Elsinore & 365 & 388 & 3622.1 & 3857.5 & $\begin{array}{c}3(365->368) 5(369->384) \\
4(385->388)\end{array}$ \\
\hline Imperial Valley & 389 & 406 & 3857.5 & 4020.0 & 30 \\
\hline Laguna Salada & 407 & 416 & 4020.0 & 4118.5 & 4 \\
\hline Garlock & 417 & 440 & 4118.5 & 4353.0 & $-5(417->426)-7(427->440)$ \\
\hline Palos Verdes & 441 & 447 & 4353.0 & 4428.6 & 3 \\
\hline Santa Cruz Island & 448 & 452 & 4428.6 & 4481.9 & -3 \\
\hline Brawley & 453 & 457 & 4481.9 & 4533.8 & 25 \\
\hline Santa Monica & 458 & 468 & 4533.8 & 4653.3 & -3 \\
\hline Cleghorn & 469 & 470 & 4653.3 & 4676.4 & -3 \\
\hline Tunnel Ridge & 471 & 472 & 4676.4 & 4695.6 & -1.3 \\
\hline Helendale & 473 & 481 & 4695.6 & 4781.7 & .8 \\
\hline Lenwood-Lockhart & 482 & 499 & 4781.7 & 4955.2 & .8 \\
\hline Pipes Canyon & 500 & 501 & 4955.2 & 4970.8 & .7 \\
\hline Gravel Hills-Harper & 502 & 509 & 4970.8 & 5051.2 & .9 \\
\hline Blackwater & 510 & 516 & 5051.2 & 5113.0 & 2 \\
\hline Camp Rock-Emerson & 517 & 527 & 5113.0 & 5227.2 & $1(517->524) .6(525->527)$ \\
\hline Homestead Valley & 528 & 530 & 5227.2 & 5254.4 & 6 \\
\hline Johnson Valley & 531 & 536 & 5254.4 & 5320.4 & .6 \\
\hline
\end{tabular}


Table 1. (continued).

\begin{tabular}{|c|c|c|c|c|c|}
\hline \multirow{2}{*}{$\begin{array}{l}\text { Fault or Fault } \\
\text { System Name }\end{array}$} & \multicolumn{2}{|c|}{ Segment Nos. } & \multicolumn{2}{|c|}{ Chart Distance $(\mathrm{km})$} & \multirow[t]{2}{*}{ Average Slip Rate (mm/yr) } \\
\hline & Begin & End & Begin & End & \\
\hline Calico-Hidalgo & 537 & 549 & 5320.4 & 5455.5 & $\begin{array}{c}1(537) 1.7(538) 2.6(539->545) \\
.6(546->549)\end{array}$ \\
\hline Pisgah-Bullion & 550 & 562 & 5455.5 & 5571.2 & 1 \\
\hline Mesquite Lake & 563 & 564 & 5571.2 & 5592.2 & 1 \\
\hline Pinto Mountain & 565 & 573 & 5592.2 & 5676.0 & -1 \\
\hline Morongo Valley & 574 & 574 & 5676.0 & 5690.6 & -.5 \\
\hline Burnt Mountain & 575 & 576 & 5690.6 & 5707.6 & 6 \\
\hline Eureka Peak & 577 & 578 & 5707.6 & 5725.8 & 6 \\
\hline Hollywood-Raymond & 579 & 582 & 5725.8 & 5763.7 & $-1(579->580)-.5(581->582)$ \\
\hline Inglewood-Rose Cyn & 583 & 604 & 5763.7 & 5979.2 & $1(583->590) 1.5(591->604)$ \\
\hline Coronado Bank & 605 & 623 & 5979.2 & 6179.5 & 3 \\
\hline San Gabriel & 624 & 637 & 6179.5 & 6310.8 & $\begin{array}{c}3(624->628) 2(630->633) \\
1(634->637)\end{array}$ \\
\hline Big Pine & 638 & 644 & 6310.8 & 6379.5 & -4 \\
\hline White Wolf & 645 & 649 & 6379.5 & 6427.6 & -5 \\
\hline
\end{tabular}

and Sykes, (1997), which contains all southern California faults with slip rates of at least $3 \mathrm{~mm} / \mathrm{yr}$. The faults are split into individual, straight segments, each of which historically often fails as a unit. Other fault parameters were taken from the table of values compiled by Barnhard and Hanson for the USGS 1996 Hazard Maps, found at http://geohazards.cr.usgs.gov/eq/faults/fsrpage01.html. Further details of construction for this instantiation of the Virtual California model will be provided elsewhere (Rundle et al., 2003). Table 1 shows the faults that are used in the model, and identifies the segments associated with them. One important fact to note is that all fault segments in the model extend from the surface to $15 \mathrm{~km}$ depth, and all are approximately $10 \mathrm{~km}$ in length along strike as described above. Thus the model uses fully three-dimensional elasticity. Slip on the segments is constant over each segment, but depth dependent slip will be examined in future models currently under development.

In the results presented below, we examined two types of failure physics, to determine the effects that can be seen on the Gutenberg-Richter magnitude frequency relation. These two types are I, dynamic fracture weakening (Eq. 12) is used only on the San Andreas fault proper, both northern and southern California branches; and II, dynamic fracture weakening is used for all faults in the model. The first type, dynamic weakening on only the San Andreas, may be of interest under the hypothesis that the most dominant fault in the system, the fault that ruptures most frequently in the largest events, has a different type of rupture physics than other faults. Note that for both models, all 650 interacting fault segments are present. Also in both types, the $C F F(x, t)=0$ failure physics, and the $\alpha$-stress leakage effect are operative. For all models examined, we take $\alpha \approx .1 / T_{R}$ for most fault segments, where $T_{R}$ is the recurrence interval that would be observed on the individual $10 \mathrm{~km} \times 15 \mathrm{~km}$ fault segment if it were in isolation (i.e., not interacting with other faults):

$$
T_{R} \equiv \frac{\sigma^{F}-\sigma^{R}}{V K_{T}}
$$

where $K_{T}$ represents the diagonal (self-interaction) term for the shear stress Green's function in the discrete representation. The exception is that $\alpha \approx .45 / T_{R}$ for the northern branch of the San Andreas fault, where we have found that the geometric complexity of the model seems to inhibit the occurrence of the large earthquakes that are observed to occur there in nature.

An example of the Coulomb Failure Function stress $C F F(x, t)$ as defined in Eq. (9) is shown in Fig. 2 for a simulation with a model of type II. The ordinate is time in years, and the abscissa is the "chart distance" (see Table 1) of each individual segment. The figure is essentially a snapshot of the dynamics. Horizontal lines represent earthquakes, and the buildup and release of $C F F(x, t)$ during the earthquake cycle can be seen in space and time. Darker colors represent lower $C F F(\boldsymbol{x}, t)$, and lighter colors represent higher $C F F(\boldsymbol{x}, t)$.

Two examples of typical large earthquakes on the northern and southern San Andreas fault are shown in Figs. 3 and 4. Note particularly that the segments participating in the event are not entirely contiguous, but that there are smaller, discontinuous groups of slipped segments participating in the event as well. The epicentral segment is shown as a darker rectangle in both figures. The earthquakes shown in Figs. 3 and 4 are taken from a model of type II.

Statistics. Figures 5 and 6 show the Gutenberg-Richter (GR) magnitude-frequency relation, with Fig. 5 associated with physics of type I (dynamic weakening on San Andreas only), and Fig. 6 associated with physics of type II (dynamic weakening on all faults). The magnitude $m$ is defined in terms of the seismic moment $M$ in the usual way:

$$
M=\mu \int s(\boldsymbol{x}, t) d \boldsymbol{x}
$$



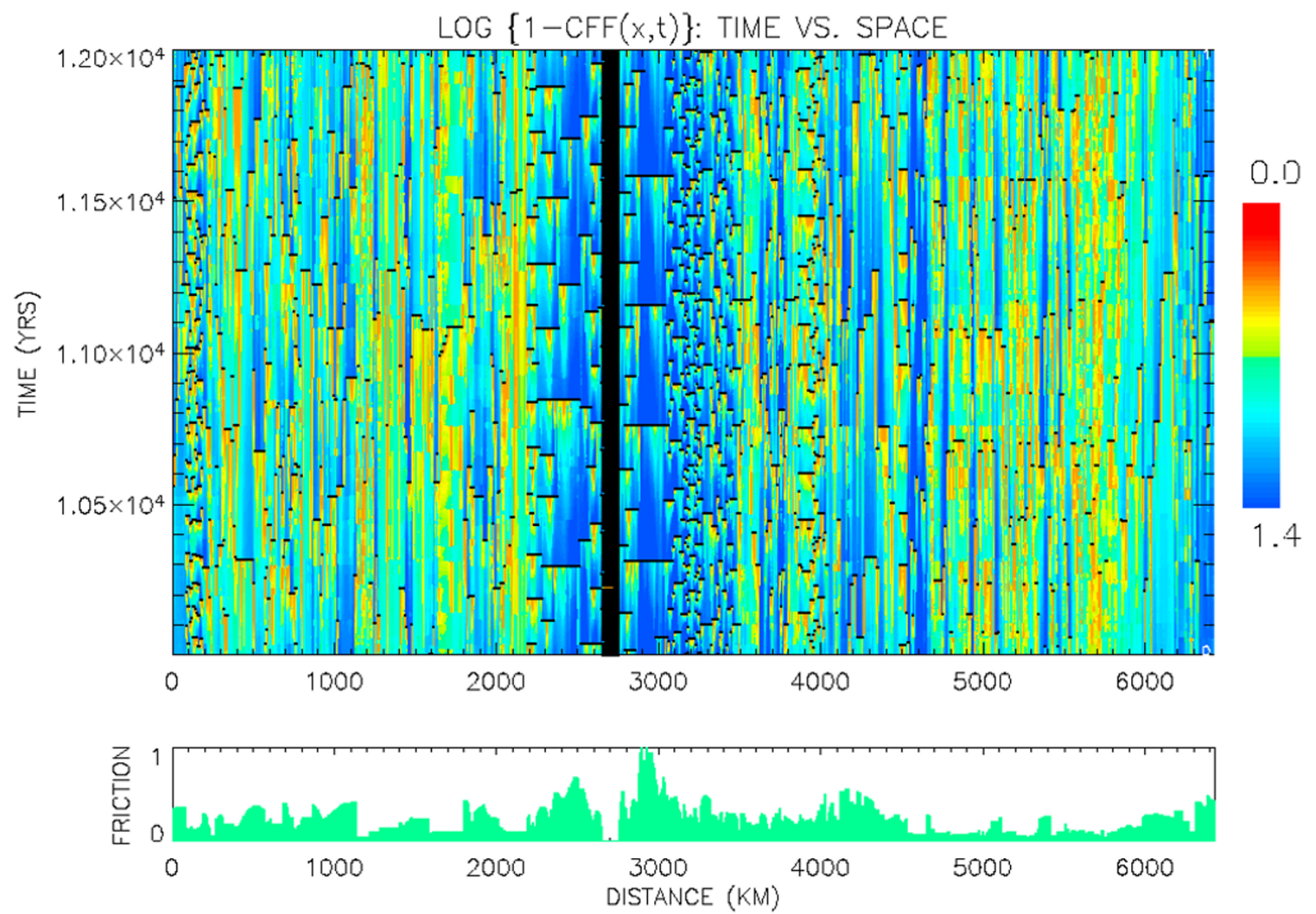

Fig. 2. Plot of Coulomb Failure Function for a time interval of 1000 years for a typical model run for a model of type II, in which all faults can dynamically weaken. Specifically, color contours of the function $\log _{10}\{1-C F F(\boldsymbol{x}, t)\}$ are plotted as a function of chart distance (see Table 1). Cool colors (low CFF stress, farther from failure) represent larger values of $|C F F(x, t)|$, and hotter colors represent smaller values (high CFF stress, closer to failure). Time is along the vertical axis and chart distance is along the horizontal axis. The bottom histogram ("Friction") is a plot of the difference between static and kinetic friction coefficients, $\mu_{S}-\mu_{K}$, as a function of (chart) distance in $\mathrm{km}$.

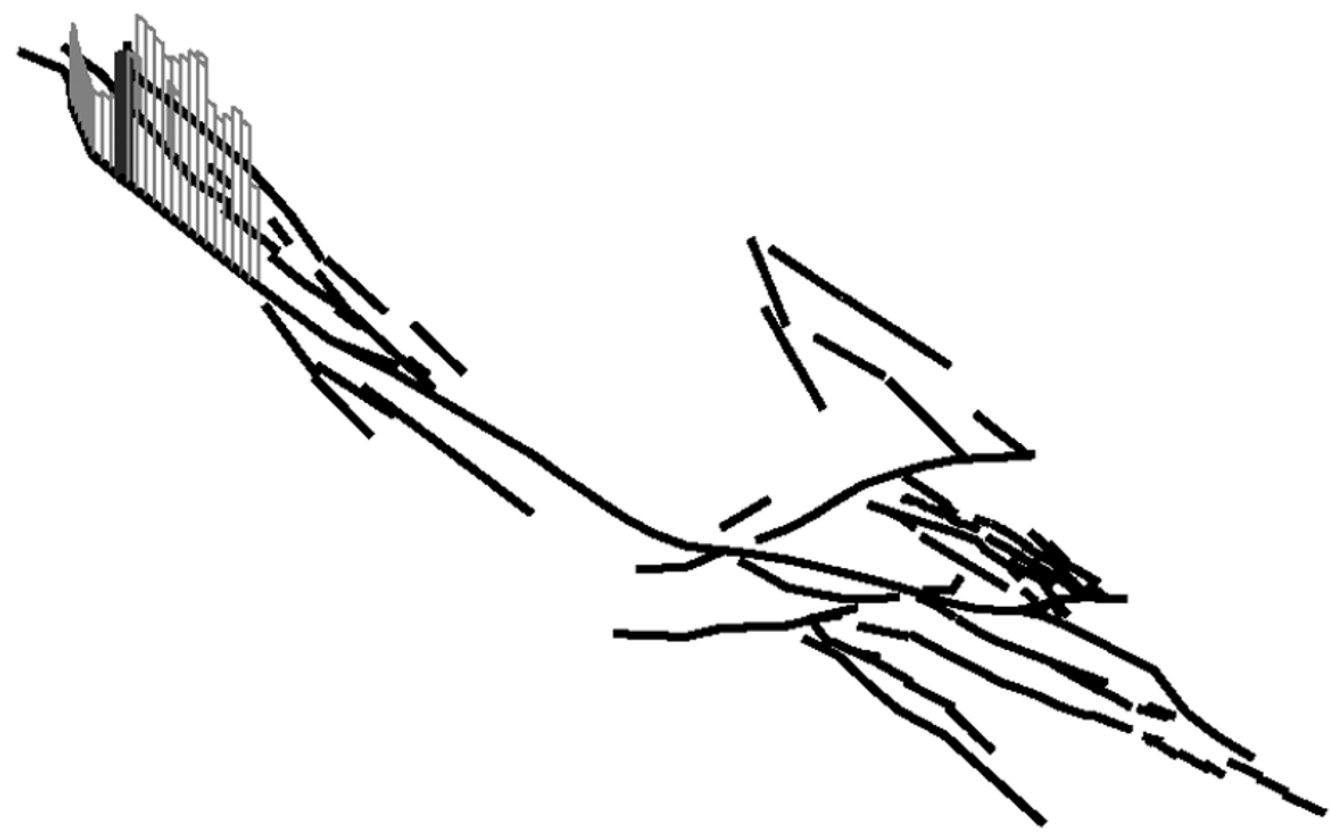

Fig. 3. Example of a large event on the northern San Andreas fault in a model of type II, in which all faults have the property of dynamic fracture weakening. The epicentral segment is shown in dark. Note that slip in this event occurs not only on the San Andreas fault proper, but also on other sub-parallel faults, demonstrating that earthquakes in the simulations are non-compact events. Maximum slip in this event is $11.04 \mathrm{~m}$. 


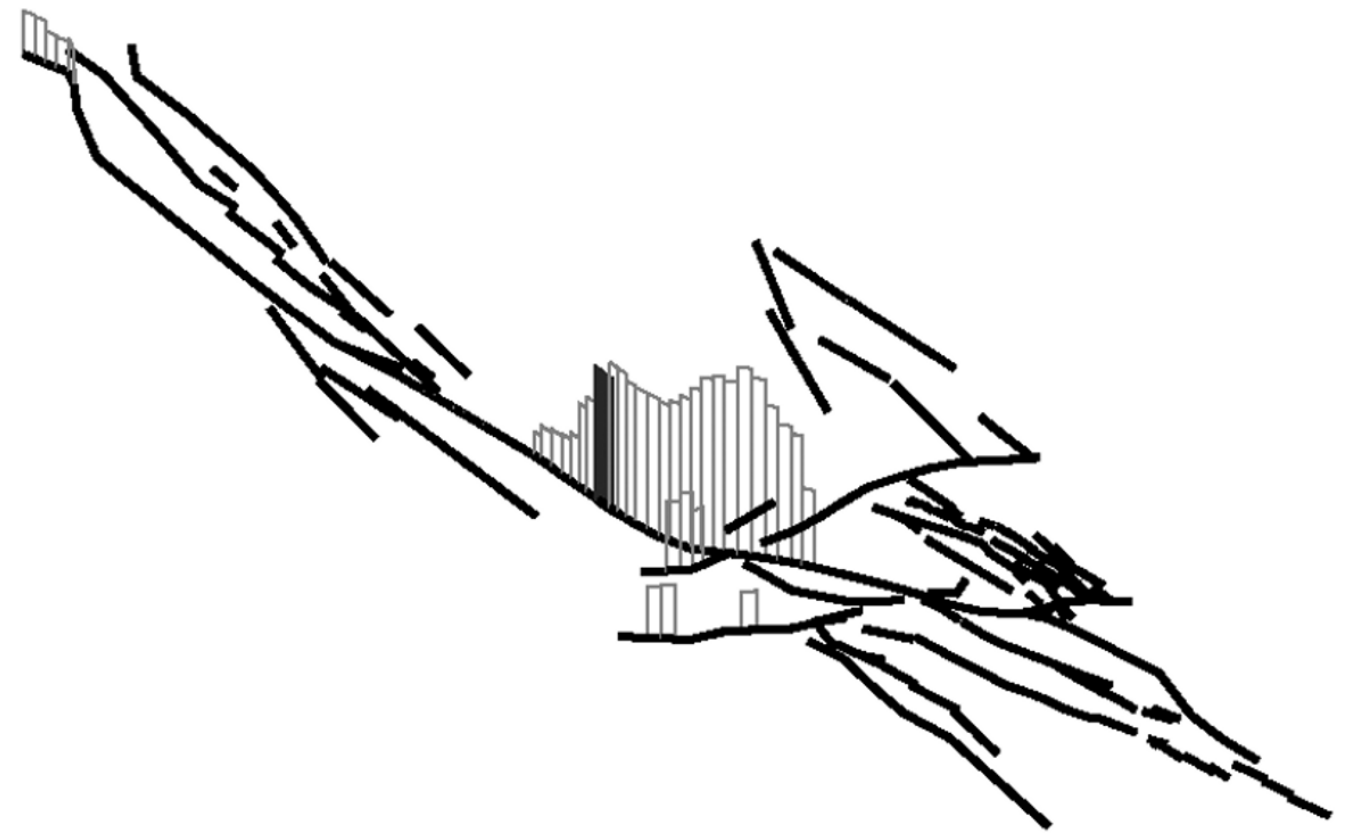

Fig. 4. Example of a large event on the southern San Andreas fault in a model of type II, in which all faults have the property of dynamic fracture weakening. The epicentral segment is shown in dark. Note that slip in this event occurs not only on the San Andreas fault proper, but also on other faults, some at considerable distance away in on the northern San Andreas, again demonstrating that earthquakes are non-compact events. Maximum slip in this event is $14.82 \mathrm{~m}$.

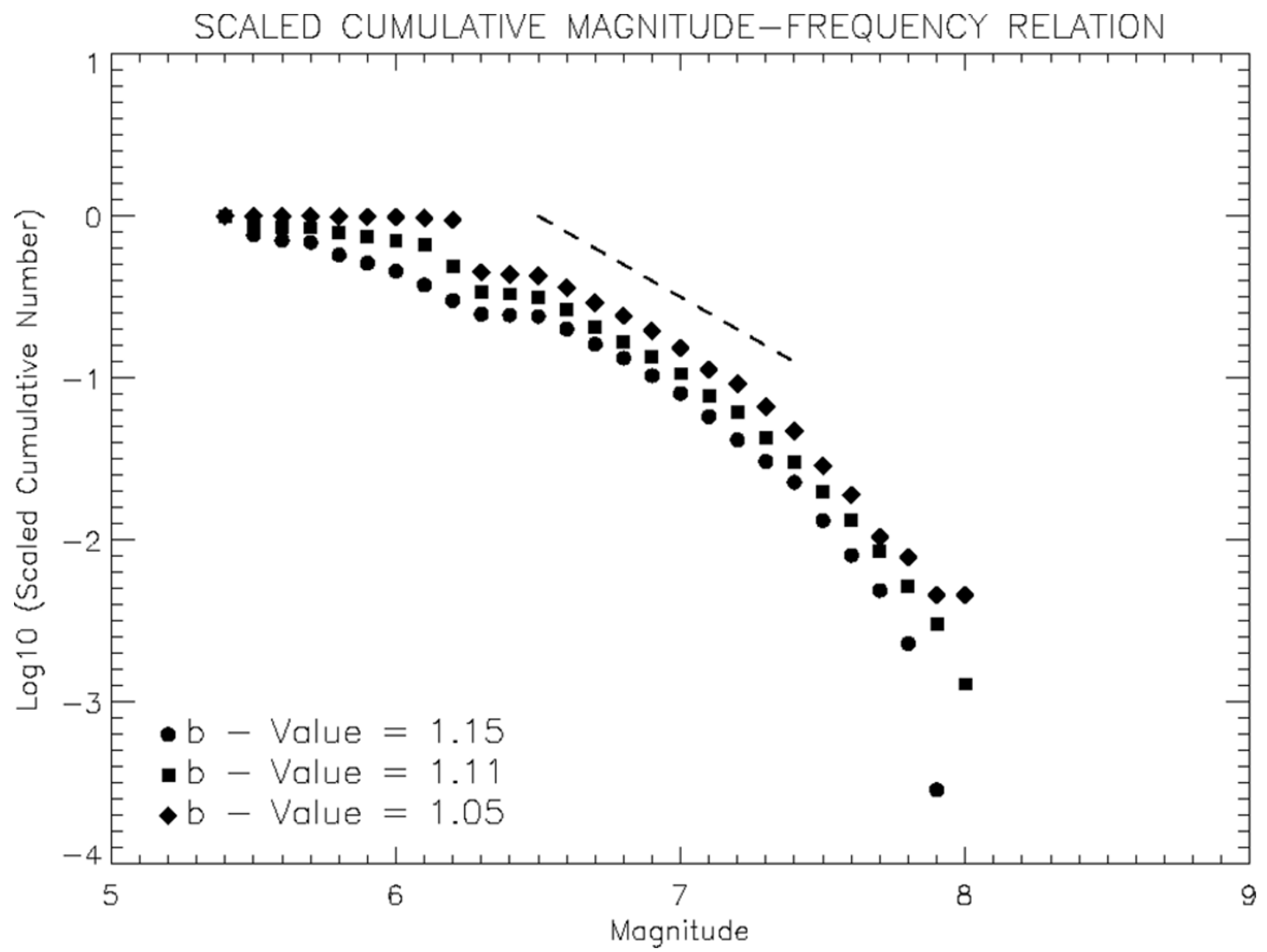

Fig. 5. Examples of 3 normalized Gutenberg-Richter curves for a model of type I. Dashed line has slope -1 and is drawn in the interval $6.5<m<7.5$. $b$-values shown are the result of fits to the points in the same interval. 


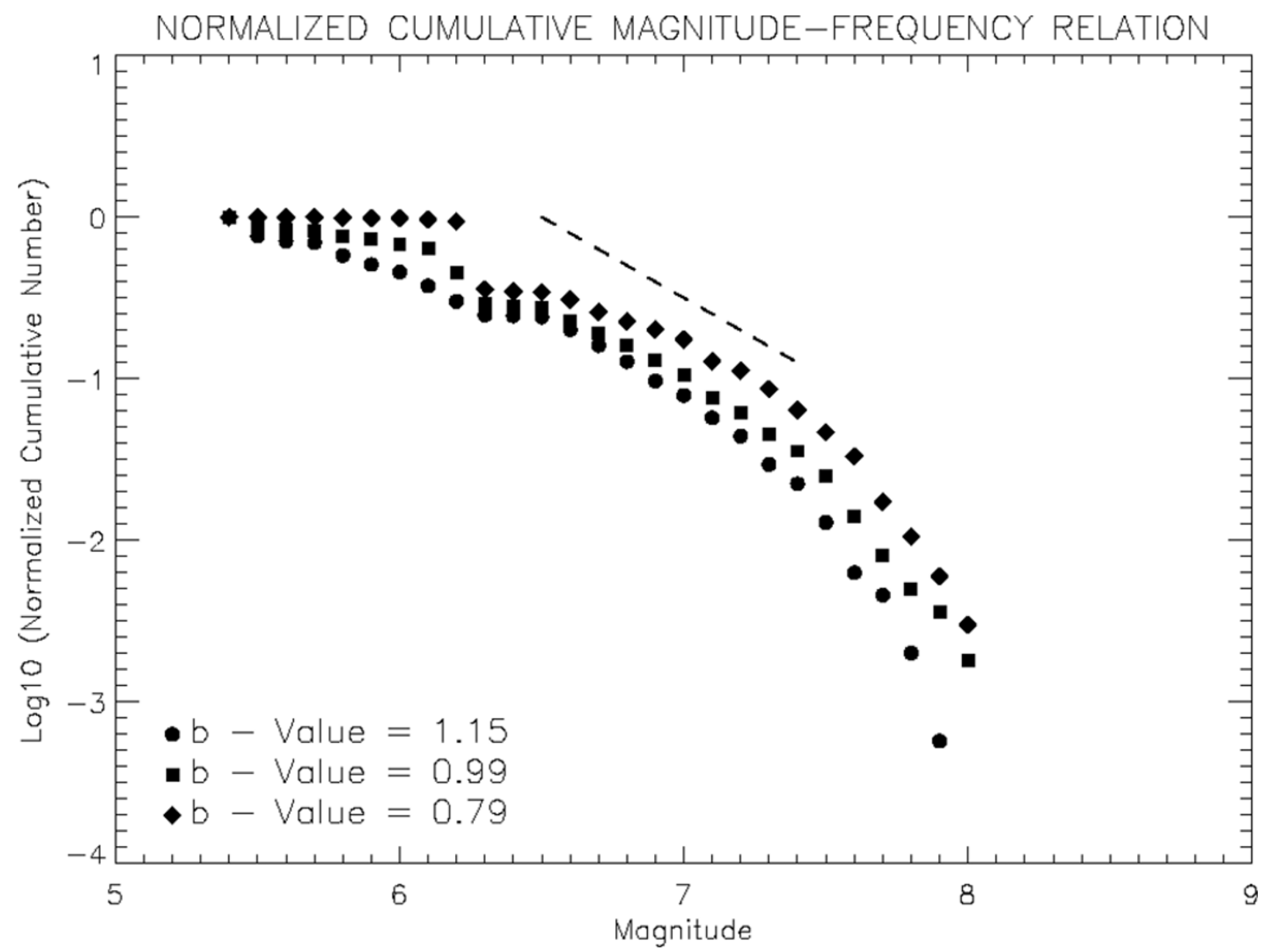

Fig. 6. Examples of 3 normalized Gutenberg-Richter curves for a model of type II. Dashed line has slope -1 and is drawn in the interval $6.5<m<7.5$. $b$-values shown are the result of fits to the points in the same interval.

$$
m=\frac{2}{3} \log _{10} M-6.0
$$

where $s(\boldsymbol{x}, t)$ is the slip at $\boldsymbol{x}$ at time $t, \mu$ is the shear modulus, and the integral is taken over all fault segments that slipped in the event at time $t$. The constant 6.0 is appropriate for variables in SI units.

It should be noticed first that the form of the GR relation (which is not well-represented by a straight line here) is strongly influenced by the minimum scale of fault segments in the model. The area of these segments is approximately $10 \mathrm{~km}$ (length) $\times 15 \mathrm{~km}$ (depth), corresponding roughly to a $m \sim 6$ earthquake. It is for that reason that a breakdown in scaling at about the $m \sim 6$ level is seen in both Figs. 5 and 6. At the other end, a cutoff of events is seen about $m \sim 8$, similar to observations in nature. In each plot, the filled circles correspond to simulations of 2000 years, having $\eta=1$; the filled squares to simulations having $\eta=.75$; and the filled diamonds to simulations having $\eta=.5$. In each figure, there is also a dashed line of slope $=1$ drawn in the range between $6.5<m<7.5$ for comparison with the points. Gutenberg-Richter $b$-values determined by fits to the curves corresponding to each symbol are given on the figure, and all are near the observed value of $b \sim 1$.

The various GR curves are all normalized, i.e., we plot the cumulative number $N(>m) / N(>-\infty)$. In Fig. 5, the total number of events is 3475 for $\eta=1$ (circles); 2323 for $\eta=.75$ (squares); and 1529 for $\eta=.5$ (diamonds). In Fig. 6, the total number of events is 3488 for $\eta=1$ (circles); 2216 for $\eta=.75$ (squares); and 1330 for $\eta=.5$ (diamonds). These numbers confirm the obvious conclusion that the physics corresponding to dynamic weakening with $\eta<1$ allows small earthquakes to grow into larger earthquakes more easily than for $\eta=1$.

From the curves shown in Figs 5 and 6, there is not a great deal of difference between the GR curves with dynamic weakening on all faults, as compared to dynamic weakening on only the San Andreas fault. The lone exception is at $\eta=.5$ where the effect is greatly magnified for the case of weakening on all faults. Finally, it can be easily seen that smaller values of $\eta$ lead to significant increases in the number of large earthquakes, with a corresponding depletion in the number of smaller earthquakes. We may presume that if there were no lower limit on earthquake size, the depletion of events near $m \sim 6$ would be compensated by smaller events that coalesce into larger events.

Acknowledgments. The authors would like to thank S. N. Ward and M. Hashimoto for careful and thoughtful reviews. This work has been supported by a grant from US Department of Energy, Office of Basic Energy Sciences to the University of California, Davis DE-FG03-95ER14499 (JBR; PBR); and under additional funding from National Aeronautics and Space Administration under grants to the Jet Propulsion Laboratory, the University of Cali- 
fornia, Davis, and the University of Indiana (JBR; PBR; AD; GCF).

\section{References}

ACES home page, http:/quakes.earth.uq.edu.au/ACES/

Earthscope home page, http://www.earthscope.org/

Deng, J. S. and L. R. Sykes, Evolution of the stress field in southern California and triggering of moderate sized earthquakes: A 200-year perspective, J. Geophys. Res., 102, 9859-9886, 1997.

Freund, L. B., Dynamic Fracture Mechanics, Cambridge University Press, Cambridge, UK 1990.

GEM home page, http://geodynamics.jpl.nasa.gov/

Gouyet, J.-F., Physics and Fractal Structures, Springer-Verlag, Berlin, 1996.

Hashimoto, M., Complexity in the recurrence of large earthquakes in southwest Japan: A simulation with an interacting fault system model, Earth Planets and Space, 52, 249-259, 2001.

Kanninen, M. F. and C. H. Popelar, Advanced Fracture Mechanics, Oxford Engineering Series 15, Oxford University Press, New York, 1985.

Karner, S. L. and C. Marone, Effects of loading rate and normal stress on stress drop and stick-slip recurrence interval, in GeoComplexity and the Physics of Earthquakes, edited by J. B. Rundle, D. L. Turcotte and W. Klein, pp. 187-198, Geophysical Monograph 120, American Geophysical Union, Washington, DC, 2000.

Matsu'ura, M., K. Nakajima, and P. Mora, eds., Proceedings of the 2nd ACES Workshop, published by APEC Cooperation for Earthquake Simulation, Tokyo and Hakone, Japan, 2001.

Mora, P., ed., Proceedings of the 1st ACES Workshop, published by APEC Cooperation for Earthquake Simulation, Brisbane, Queensland, AU, 1999.

Nature debate on earthquake forecasting: http://www.nature.com/nature/ debates/earthquake/equake/frameset.html, 1999.

Preston, E. L., Abelian Fault Models, Ph.D. dissertation, University of Colorado, 2001.

Rabinowicz, E., Friction and Wear of Materials, John Wiley, New York, 2nd ed., 1995.

Rundle, J. B., A physical model for earthquakes, 2. Application to southern California, J. Geophys. Res., 93, 6255-6274, 1988

Rundle, J. B., D. L. Turcotte, and W. Klein, eds., Geocomplexity and the Physics of Earthquakes, Geophysical Monograph 120, American Geophysical Union, Washington, DC, 2000a.
Rundle, P. B., J. B. Rundle, K. F. Tiampo, J. S. S. Martins, S. McGinnis, and W. Klein, Triggering dynamics on earthquake fault networks, in Proc. 3rd Conf. Tectonic Problems of the San Andreas Fault System, edited by G. Bokelmann and R. L. Kovach, pp. 305-317, Stanford U. Publ., Geol. Sci., XXI, 2000b.

Rundle, P. B., J. B. Rundle, K. F. Tiampo, J. S. S. Martins, S. McGinnis, and W. Klein, Nonlinear network dynamics on earthquake fault systems, Phys. Rev. Lett., 87, 148501(1-4), 2001.

Rundle, J. B., K. F. Tiampo, W. Klein, and J. S. S. Martins, Self-organization in leaky threshold systems: The influence of near-mean field dynamics and its implications for earthquakes, neurobiology, and forecasting, Proc. Nat. Acad. Sci., 99, suppl., 2514-2521, 2002.

Rundle, J. B., P. B. Rundle, and A. Donnellan, Statistics and stress evolution in simulations of Virtual California 2001, manuscript in preparation, 2003

Saxena, A., Nonlinear Fracture Mechanics foe Engineers, CRC Press, Boca Raton, FL 1998.

SCEC home page, Southern California Earthquake Center, http://www.scec.org/

Scholz, C. H., The Mechanics of Earthquakes and Faulting, Cambridge University Press, Cambridge, UK, 1990.

Stein, S. and A. Newman, Characteristic and uncharacteristic earthquakes as possible artifacts: Applications to the New Madrid and Wabash seismic zones, Seism. Res. Lett., 75, 173-187, 2004.

Tullis, T. E., Rock friction and its implications for earthquake prediction examined via models of Parkfield earthquakes, Proc. Nat. Acad.Sci. USA, 93, 3803-3810, 1996.

Turcotte, D. L., Earthquake prediction, An. Rev. Earth. Planet. Sci., 19, 263281, 1991.

Vicsek, T., Fractal Growth Phenomena, World Scientific, Singapore, 1989.

Ward, S. N., San Francisco bay area earthquake simulations, a step towards a standard physical model, Bull. Seism. Soc. Am., 90, 370-386, 2000.

Zoback, M. L., 1st-order and 2nd-order patterns of stress in the lithosphere-The World Stress Map project, J. Geophys. Res., 97, 11703$11728,1992$.

J. B. Rundle (e-mail: rundle@physics.ucdavis.edu), P. B. Rundle, A. Donnellan, and G. Fox 